\title{
Outcomes of a Cross-Disciplinary Concussion Prevention and Diagnosis Workshop Series ${ }^{\dagger}$
}

\author{
Patrick Drane ${ }^{1, *}$, Xingwei Wang ${ }^{2}$, Murat Inalpolat ${ }^{3}$ and James Sherwood ${ }^{1}$ \\ 1 Baseball Research Center, University of Massachusetts Lowell, 1 University Avenue, Lowell, MA 01854, \\ USA; James_Sherwood@uml.edu \\ 2 Electrical Engineering Department, University of Massachusetts Lowell, 1 University Avenue, Lowell, \\ 01854, USA; Xingwei_Wang@uml.edu \\ 3 Mechanical Engineering Department, University of Massachusetts Lowell, 1 University Avenue, Lowell, \\ MA 01854, USA; Murat_Inalpolat@uml.edu \\ * Correspondence: Patrick_Drane@uml.edu; Tel: +1-(978)-934-2996 \\ + Presented at the 12th Conference of the International Sports Engineering Association, Brisbane, \\ Queensland, Australia, 26-29 March 2018.
}

Published: 25 February 2018

\begin{abstract}
This paper reports the outcomes of a series of two Concussion Research Workshops held in Lowell, MA, USA. The workshop examined the state-of-the-art in concussion research, research challenges and the future directions of research within the following three core topic areas: (A) Concussion Prevention Techniques \& Technology, (B) Concussion Diagnosis, and (C) Treatment of Concussions. Concussions are a form of traumatic brain injury caused by an impact and are a growing concern among athletes and those who are involved with sports. Recent years have led to increasing awareness and research related to concussions with limited definitive understanding of the specific mechanism and pathology. Technology is beginning to take on an important role in the prevention, diagnosis and treatment of concussions. Currently, sensors provide data about the impact and the athlete. However, sensors and better protective equipment can enable an effective monitoring and thus protection of athletes. Only when a more definitive understanding of the injury mechanism is achieved, can sensors and protective equipment design contribute to effective monitoring and protection of athletes.
\end{abstract}

Keywords: concussion; workshop; interdisciplinary; helmet; acceleration; TBI

\section{Introduction}

Concussion prevention and diagnosis is at the forefront of the efforts of many medical personnel, engineers and scientists. Yet, there is still too much unknown about concussions to effectively prevent, diagnose and treat them. In recent years, there has been a significant upsurge in the awareness surrounding concussions. This awareness offers important progress in education of the public, and some increase in the desire for understanding and research funding. However, the increase in awareness has not led to a clear understanding of concussions and how they occur yet. Although research is being performed in a variety of disciplines, there are gaps between these research efforts causing potential solutions and comprehensive understanding to remain elusive.

The University of Massachusetts Lowell hosted two interdisciplinary workshops in 2015 and 2017 , both focusing on the identification and discussion of the gaps between concussion prevention and diagnosis efforts among the diverse participants. Panelists were selected to reflect a diversity of backgrounds and expertise. Small-group discussions focused on questions targeted on identifying the gaps in research, especially research which interweaves between the broad areas within the disciplines. Three primary issues were identified during the 2015 workshop, (1) the need for greater 
collaboration and communication between the disciplines working in this research area; (2) a continued general lack of clinical understanding of the mechanism of concussions; and (3) the opportunity for the development of better protective equipment (headgear) and better training techniques. Many of these preventative measures can only be adequately implemented following a better understanding of the source of concussions.

Concussions are a form of traumatic brain injury that affects brain functions and usually caused by a blow to the head, but can also be caused by violently shaking the head and upper body. Some concussions can cause loss of consciousness, but most do not. Most people usually recover fully after a concussion [1]. However, repetitive head impacts and trauma have been linked to serious health consequences ranging from learning challenges in collegiate athletes to the development of chronic traumatic encephalopathy (CTE), in which neurofibrillary tangles throughout the brain $[2,3]$. With the importance of avoiding concussion causing impacts, it is necessary that the athletes and the public are aware of the causes and effects of concussions. Therefore, wide-spread education is critical. An evaluation of the Sports Legacy Institute Community Educators (SLICE) curriculum showed significant improvements in knowledge about concussion recognition among various youth populations [4]. Even with improving awareness, the actual rates of diagnosing concussions in youth athletes are low. Therefore, there is a need for objective and qualitative diagnostic tools to assist in the diagnosis. However, current technologies have been found to be generally unreliable for aiding clinical utility [5]. There are many current research and development efforts being undertaken to create advanced technologies that will likely rely on the rapid developments of wearable sensors being developed for a wide variety of patient, athlete and everyday monitoring conditions [6].

\section{Workshop Structure}

Both workshops took place over two days and each was attended by 40+ participants from various professional backgrounds and expertise, including engineering, computer science, medicine, athletic training, neuroimaging, and personal experiences with concussions. Both days began with keynote talks to provide an expert overview of specific research fields of broad importance to advances in concussion research. Figure 1 shows a flow chart of the workshop structure and approach and the links between the 2015 and the 2017 workshops.

In the 2017 workshop, day one started off with a keynote talk by Dr. Stefan Duma on advances in the design and testing of helmets. Next, three panel sessions were held to provide a high level overview of critical elements of the cross disciplinary nature of this research. Each panelist provided an overview of their expert perspective on the topics of getting started on concussion research, the role of humans in concussion research, and the role of data in concussion research. Several prepared questions were asked of the panel and were followed by a group question-and-answer session. At the end of the first day of the workshop, lab tours were provided to enable the participants to get a hands-on perspective of some engineering tools available for concussion research. Day two began with a keynote talk by Dr. Robert Cantu on the medical advancements in concussions and Chronic Traumatic Encephalopaty (CTE). That talk was followed by a second keynote talk by Dr. Paolo Bonato on the role of connected health and wearable technologies. Next, three panel sessions with topics on concussion prevention techniques and technology, concussion diagnosis, and treatment of concussions were held with overviews by the expert panelists allowing more focus on discussion. 


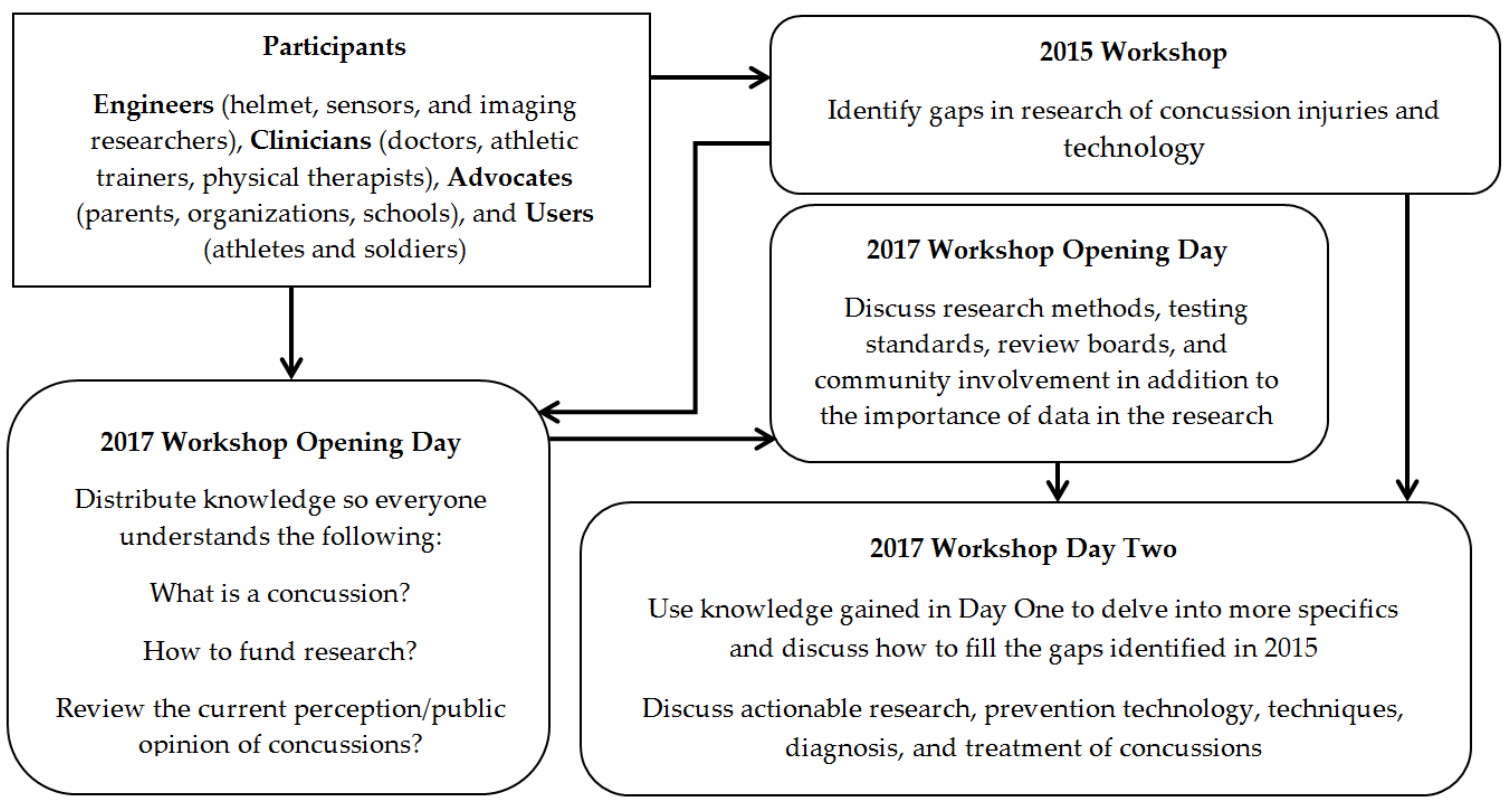

Figure 1. Flow Chart Displaying the Structure of the Workshop.

\section{Outcomes and Discussion}

\subsection{Keynote Talks}

Three keynote talks were given during the workshop. The first talk provided a history and advances in the design and testing of helmets. Through history helmet technologies have advanced significantly and regulations and testing have often pushed those changes. The second talk provided an overview of the medical advances in concussions and CTE. Concussions are no longer consistently classified a Mild Traumatic Brain Injury (mTBI) and there is growing consensus that at least some concussions are a form of Traumatic Brain Injury (TBI). He also stressed the importance of proper coaching techniques including having football coaches instruct youth on how to properly tackle, mitigating the chances of concussion. In addition, he mentioned that stronger enforcement of rules and penalties for taking off helmets can also help prevent concussions. One of the topics that were discussed after the talk was on how in baseball, research on injuries to players was being extended to include injuries to umpires. The third talk provided an overview of the role of connected health and wearable technologies and discussed advancements in sensor technology such as the new sensors with the ability to receive radio signals and detect mood changes and anxiety. Two different sensors with Bluetooth connectability were compared and the one easier to distribute to youth, capable of collecting data in real time, with excellent data security, and more affordable was favored.

\subsection{Getting Started on Concussion Research}

The first panel session provided an overview of concussion science, an overview of recent public opinion surveys surrounding sports concussions and CTE, and finally an overview of grants and funding mechanisms to pursue advances in concussion research. The panel reviewed the areas of physical, cognitive and sleep symptoms caused by concussions. The panelist identified that there are 3.8 million sport-related Traumatic Brain Injury (TBI) per year in the United States and that only 50\% of moderate to severe concussions are detected by an medical doctor, noted that injury to the Axonal is the most dangerous injury and is known as a neuro-metabolic concussion and that Post-Concussion Syndrome typically lasts more than 28 days and can effect neuro transmitters.

The public opinion surveys identified the concerns of parents, especially new parents, who feel that tackle football and soccer can contribute to brain injury. It was also identified that the majority of Americans would favor changing the age (increased to high school age) in which a child could play tackle football or soccer. There is desire for leagues to compile new statistics. 
The panel concluded with an overview of managing external research funding, grants, contracts and regulations. A number of federal granting agencies including the Centers for Disease Control and Prevention (CDC), the US Army Medical Research and Material Command (USAMRMC), the National Institutes of Health (NIH), and the National Science Foundation (NSF) were identified in addition to a number of not-for-profit/foundation granting agencies.

\subsection{Humans and Research}

The second panel provided an overview of research methods and requirements. First, an overview on the purpose and definitions of Institutional Review Boards (IRBs) and how they are important for protecting human subject involved in research. Each researcher needs to develop their protocol, consent statement, and consider additional factors such as community partners, scripts, and reporting adverse events.

A prospective study is a research method where data is collected as events occur; whereas, a retrospective study is a study where data that has been collected from records and patient recall. Prospective studies are often necessary, but retrospective studies are often desirable as they are less timely and less costly. Prospective cohort studies are often challenged by incomplete records, inaccurate records, and selection bias.

Next, an overview of the role of utilizing biostatistics was provided. Biostatisticians aid in designing studies, analyzing results, and interpreting and reporting those results. It is important that the evaluation of the effectiveness of an intervention be defined and therefore that enough data be collected to be able to obtain a conclusive result.

Concussion research often must include interactions with community partners. This panel identified many positive impacts of engaging the community such as local knowledge and broader community acceptance of research. The panel also identified numerous challenges for this process such as working with non-traditional communities, overcoming differences between academics and the community, and overcoming competing priorities. Knowing the community and identifying mutual benefits for both groups were identified as methods for addressing those challenges.

\subsection{The Role of Data in Concussion Research}

Data has a significant role in concussion research. More and more sensors and larger and larger studies are becoming the norm. This panel focused on the generation and security of data. First, a brief overview of sensors was provided. Two types of accelerometers (piezoelectric and capacitive), gyroscopes, fiber optic sensors, GPS receivers, fabric sensors, pressure sensors are commonly used. Rotational accelerations were identified as critically important in addition to the more traditional linear accelerations. Wireless sensors (potentially Bluetooth) are critical for the field.

Data security is important. Some concussion sensing data could potentially fall under Healthcare Insurance Portability and Accountability Act (HIPAA) or other similar laws for data security. Four policies include in HIPAA are (1) the privacy rule which dealt with who had access to medical data information; (2) the security rule which dealt with procedures for protecting that data; (3) establishing electronic health records for all Americans; and (4) promoting of standardization of medical data information.

\subsection{Prevention, Diagnosis, and Treatment Workshop Panels}

The concussion prevention panel focused its discussion on training methods which have being implemented with success. An overview of the risks associated with American football tackling and theories behind those risks were discussed. Helmetless tackling training (HuTT) is on program whose goals include improving public safety and reduction of head injuries by optimizing coaching techniques, developing age appropriate drill prescription, and improving tackling and blocking behaviors.

The concussion diagnosis panel focused its discussion on sideline concussion diagnosis and the need for objective sensors. The initial evaluation determines concussion through early symptoms and 
concussion assessment tests going from the standardized assessment of concussion to balance error scoring system. Post-concussion assessment tests such as acute concussion evaluation and simple recovery strategies. Two types of sensors, strain and movement (including angular and translational) were identified as useful.

Following the diagnosis of concussions, there is a need for treatment. In the early years, treatment was simply rest until the patient was non-symptomatic. The panel identified that new treatment strategies have become effective, but lack the prospective and longitudinal studies to confirm the effectiveness and limitations.

\section{Conclusions}

The two NSF-funding workshops were structured to bring together a highly-interdisciplinary group of researchers and practitioners to discuss the current state of concussion research and determine paths forward to address the prevalence of concussions in and out of sports. These workshops facilitated discussions about the current state of the knowledge with respect to the sources, prevention, diagnosis, and treatment of concussions and provided an opportunity for the establishment of new working teams.

New technologies are being developed and used, but there are concerns about the reliability of those technologies, in part because there are very few standards or testing for the equipment. The designs of new helmet systems must be advanced, but the helmets must be tailored to the types of impacts which cause traumatic brain injuries in the sports. The design of helmets and other protective equipment must address both rotational and linear accelerations caused by differing impact locations, magnitudes and durations and differing athlete susceptibility to those impacts. Neck strength was one physiological condition identified which could have a significant contribution and therefore could be a subject of more research.

The most significant concussion diagnosis challenge identified is lack of a definitive objective marker to confirm that the person actually experienced a concussion. It was discussed that even if such a perfect bio-marker was found, it would not conclude which section of the brain were affected. This presents a challenge for educated decision making in return-to-play and return to normal activities. Ocular response was identified as one potential method for detecting concussions. Preseason and post-impact tests often include a subjective combination of balance, eye movement, and cognitive tests to diagnosis a concussion. Concussion diagnosis is also plagued by concerns of under-reporting of concussions and concussive symptoms in sports.

One of the most significant concussion treatment research gaps is the lack of double-blind studies to evaluate the current treatment practices. Currently, rest is the only treatment commonly recommended, and this is partly because it is known that rest cannot aggravate the symptoms. Light cognitive exercise now sometimes used to treat concussions so long as it does not exacerbate the symptoms. In addition to affecting the initial impact, factors like nutrition can also affect the healing process after an impact. Treating concussion with nutritional supplements, such as Zinc, was identified as a potential treatment method, but those treatments lack the necessary clinical studies to determine efficacy. Return-to-play, study, and activity guidelines also lack the research to have clinical confidence in those practices.

The 2017 workshop focused on facilitating each participant's basic knowledge about the crossdisciplinary elements of advancing concussion research. Key elements of advances in wearable and imaging technologies were discussed and identified as critical areas of research which offer abilities to contribute to overcoming the lack of understanding about the impact conditions. Many of the challenges to overcome are part of other broad areas of research in wearable sensors, flexible electronics, big data, active image processing, data security, and smart and connected health systems.

Acknowledgments: This material is based upon work supported by the National Science Foundation under Grant No. 1540100. Any opinions, findings, and conclusions or recommendations expressed in this material are those of the author(s) and do not necessarily reflect the views of the National Science Foundation. The workshop was also funded through paid registrations by some of the participants. The authors acknowledge all of the speakers who contributed to the workshop: Robert Cantu, Paolo Bonato, Stefan Duma, J. Herbert Stevenson, 
Naseem Chatiwala, Jay Myers, John Cluverius, Anne Maglia, Elaine Major; Rebecca Gore, David Turcotte, JoAnn Moriarty-Baron, Elena Bannerman, Kimberly Sikule, James A. DeProfio; and Paul Cuetara.

Conflicts of Interest: The authors declare no conflict of interest. The funding sponsors had no role in the design of the study; in the collection, analyses, or interpretation of data; in the writing of the manuscript, and in the decision to publish the results.

\section{References}

1. Mayo Clinic. Available online: www.mayoclinic.org/diseases-conditions/concussion/home/ovc-20273153 (accessed on 26 September 2017).

2. McAllister, T.W.; Flashman, L.A.; Maerlender, A.; Greenwald, R.M.; Beckwith, J.G.; Tosteson, T.D.; Crisco, J.J.; Brolinson, P.G.; Duma, S.M.; Duhaime, A.C.; et al. Cognitive effects of one season of head impacts in a cohort of collegiate contact sport athletes. Neurology 2012, 78, 1777-1784, doi:10.1212/WNL.0b013e3182582fe7.

3. McKee, A.C.; Gavett, B.E.; Stern, R.A.; Nowinski, C.J.; Cantu, R.C.; Kowall, N.W.; Perl, D.P.; Hedley-Whyte, E.T.; Price, B.; Sullivan, C.; et al. TDP-43 Proteinopathy and Motor Neuron Disease in Chronic Traumatic Encephalopathy. J. Neuropathol. Exp. Neurol. 2010, 69, 918-929, doi:10.1097/NEN.0b013e3181ee7d85.

4. Bagley, A.F.; Daneshvar, D.H.; Schanker, B.D.; Zurakowski, D.; d'Hemecourt, C.A.; Nowinski, C.J.; Cantu, R.C.; Goulet, K. Effectiveness of the SLICE Program for Youth Concussion Education. Clin. J. Sport Med. 2012, 22, 385-389, doi:10.1097/JSM.0b013e3182639bb4.

5. O'Connor, K.L.; Rowson, S.; Duma, S.M.; Broglio, S.P. Head-Impact-Measurement Devices: A Systematic Review. J. Athl. Train. 2017, 52, 206-227, doi:10.4085/1062-6050.52.2.05.

6. Daneault, J.-F.; Kanzler, C.; Lee, S.; Golabchi, F.; Vergara-Diaz, G.; Carvalho, G.F.; Fabara, E.; Sapienza, S.; Sudarsky, L.; Growdon, J.; et al. Exploring the use of wearable sensors to monitor drug response of patients with Parkinson's disease in the home setting. Neurology 2017, 88, P4.002.

(C) 2018 by the authors. Licensee MDPI, Basel, Switzerland. This article is an open access article distributed under the terms and conditions of the Creative Commons Attribution (CC BY) license (http://creativecommons.org/licenses/by/4.0/). 